\title{
Prospective Interventional Non-Comparative Non-Randomized Clinical Study of A Topical Synergic Compound to Mitigate Presbyopia Effects
}

Cesar A Sanchez Galeana ( $\sim$ optallvision@gmail.com )

Optall Vision https://orcid.org/0000-0001-8064-4858

\section{Research Article}

Keywords: topical synergic compound, mitigate presbyopia effects, pilocarpine, brimonidine, oxymetazoline

Posted Date: December 21st, 2021

DOl: https://doi.org/10.21203/rs.3.rs-1005080/v1

License: (1) (1) This work is licensed under a Creative Commons Attribution 4.0 International License. Read Full License 


\section{Abstract}

Purpose To assess the safety and efficacy of a novel compound with low -dose pilocarpine, brimonidine and oxymetazoline (PBO) in the near vision of a group of healthy presbyopic individuals.

Design Prospective, consecutive, noncomparative, nonrandomized interventional study.

Subjects Twenty-six healthy presbyopic patients presented from August to September 2021 to our clinic and were willing to participate in the study.

Methods PBO compound was instilled in both eyes to assess its effect in near vision one hour after instillation.

Main Outcome Measures Under corrected near visual acuity using the Jaeger notation, under corrected and corrected distance visual acuity, photopic and scotopic pupil diameter was registered before and one hour after instillation of the compound.

Results Baseline binocular Jaeger notation was 5.86 +/-1.39 SD range (3-9). One hour after drop instillation, binocular Jaeger was $2.53+/ 1.71$ SD range (1 $6)(p \leq 0.0001)$ Lines gained $3.30+/-1.40 \mathrm{SD}$ range $(0-6)$. Mean photopic pupilar diameter was $3.69 \mathrm{~mm}+/-0.67 \mathrm{SD}$ range $(2.5-5 \mathrm{~mm})$. Mean scotopic pupilar diameter was $4.61 \mathrm{~mm}+/-0.78 \mathrm{SD}$ range $(3-6 \mathrm{~mm})$. The mean photopic pupilar diameter was $2.27 \mathrm{~mm}+/-052 \mathrm{SD}(1.5-3)(\mathrm{p} \leq 0.000)$. Scotopic pupilar diameter was $2.63 \mathrm{~mm}+/-0.53 \mathrm{SD}$ range $(2-3)(\mathrm{p} \leq 0.000)$. One patient reported no improvement in near vision. (3.8\%). One patient reported dim vision rated 5 in a VAS $(1-10)(3.8 \%)$.

Conclusion Low-dose pilocarpine, brimonidine, and oxymetazoline showed efficacy, improving near-distance vision one hour after instillation in a group of presbyopic patients, and safety showed few and transient secondary effects.

\section{Introduction}

There is an increasing surge worldwide to address presbyopia symptoms with a pharmacological solution. Certainly, there is a niche of patients who would benefit from such innovative technologies. This technology should be capable of competing with the standard of care, such as spectacles, contact lenses, ${ }^{1}$ laser refractive technique, ${ }^{2-5}$ inlays, ${ }^{6,7}$ scleral implants, ${ }^{8}$ lens interventions with femtosecond lasers, 9 pseudoaccommodative lenses, ${ }^{10}$ multifocal intraocular lenses ${ }^{11}$ and pharmacological arrays of pilocarpine- and nonpilocarpine-based pharmacological strategies. ${ }^{12-15}$

Pilocarpine is a parasympathetic mimetic drug that binds to muscarinic receptors $\mathrm{m} 3$. It is mainly used for the treatment of narrow angle glaucoma at concentrations ranging from $1 \%$ to $4 \%$ for more than one hundred years, causing miosis, ciliary body contraction, and pulling the scleral spur, therefore lowering the intraocular pressure. ${ }^{16} \mathrm{Among}$ the side effects are ciliary spasm, myopization, red eye, eyebrow pain, and posterior iris synechiae related to chronic use. ${ }^{16,17}$ Brimonidine is an alpha 2 adrenergic agonist used for the treatment of glaucoma at concentrations from 0.15 to $0.2 \% .{ }^{20,21}$ It blocks the alpha 2 receptors of the iris dilator muscle, inhibiting its function and then inducing miosis. This side effect has been used to control nocturnal glare and halos experimented with by patients after laser excimer procedures. ${ }^{22-24}$ Oxymetazoline is an alpha 1 and partial alpha 2 adrenergic agonist used as a vasoconstrictor for the treatment of red eye and has an effect dilating the pupil due to its prejunctional alpha 2 activity leading to a reduction in sensory neurotransmitter release, as evident from a decrease in evoked sphincter contraction. Pilocarpine, brimonidine and oxymetazoline in a low-dose compound have a synergistic effect, reducing presbyopia symptoms better than pilocarpine or brimonidine alone, as demonstrated in an earlier clinical trial by our group (ClinicalTrials.gov NCT05001243).

We prepared a patent pending compound (MX/a/2020/012116) using pilocarpine, brimonidine and oxymetazoline at low doses to determine its effect on near vision and pupil diameter to ameliorate presbyopia symptoms and secondary effects related to drug use, such as pain, redness and decreased distance visual acuity. ${ }^{25}$

\section{Material And Methods}

A prospective, consecutive, interventional noncomparative, clinical trial was conducted from August to September 2021 . We recruited 26 consecutive patients presenting at the Optall Vision eye center in México City with presbyopia symptoms. The study was approved by the ethics committee of Optall Vision Eye Center in México City, following the declaration of Helsinki of 1964. All patients signed and provided informed consent to participate in the study. We included healthy patients with presbyopia. Inclusion criteria: Healthy, no prior eye surgeries, no prior eye diseases, myopia (0 - -0.50$)$ D, Hyperopia ( $1-1.50)$ D, Cylinder (0 - -1.50) D. Corrected visual acuity 20/25 or better. Presbyopia diagnosis was determined if uncorrected near vision acuity was equal to less than Jaeger 3 in the Rosenbaum chart, improving at least Jaeger 2 with the use of at least a $+1.00 \mathrm{D}$ lens. We registered age, gender, ocular dominance, uncorrected visual acuity (UCVA) measured with the Snellen chart, refraction, best corrected visual acuity (BCVA), uncorrected near vision acuity with the Rosenbaum Jaeger chart, (UNVA), best corrected near vision acuity (BNVA) Pupil diameter in photopic and scotopic conditions (PD), Biomicroscopic slit lamp exam, eye fundus exam. A compound of low-dose pilocarpine (4.3 mg) (Pil, Sophia Laboratories, México), brimonidine (0.43 mg) (Agglad, Sophia Laboratories, México), oxymetazoline/sodium hyaluronate $(2.1 \mathrm{mg} / 0.26 \mathrm{mg}$ ) (Hyalox, Sophia Laboratories, México) and bromofenac (0.13 mg) (Zebesten, Sophia Laboratories, México) was formulated in a pharmacy. One drop of PBO compound was instilled in both eyes. After one hour of uncorrected visual acuity (UCVA), best corrected visual acuity (BCVA), uncorrected near vision acuity (UNVA), and best corrected near vision acuity (BNVA), pupil diameter (PD) pain in an analogue visual scale (AVS) was measured from 0 - 10. Hyperemia was graded from 0 to 4. Primary outcome: To measure the effect of the PBO synergic compound on near vision of presbyope healthy individuals one hour after instillation. Secondary outcome: to measure pupil size change one hour after instillation of the PBO synergic compound. Statistical analysis was run with SPSS statistica using Pearson and Spearman correlation analysis and Wilcoxon nonparametric tests. $P$ value is significant when is equal or less than 0.05 . 


\section{Results}

Descriptive statistics. Twenty-six patients were recruited to participate in the study: 15 males and 11 females. Mean age was $51.86+/-6.31$ SD range (42 - 63 years). BCVA was $20 / 20$ in all eyes. Binocular Jaeger score was $5.86+/-1.39$ SD range (3 - 9). Mean sphere OD was $0.43+/-0.68 D(-0.5-1.75)$, and the mean sphere OS was $0.69+/-0.51$ Mean cylinder OD was $-0.66+/-0.51 \mathrm{D}$ range $(-0.25--1.5)$, mean cylinder OS was $-0.58+/-0.35 \mathrm{D}$ range $(-0.25--1.5)$. Mean addition OD was $1.82+/-0.55 \mathrm{D}$ range (1.00 - 3.00), mean addition OS was 1.77+/-0.54 D range (1.00 - 3.00).

Mean photopic pupilar diameter was $3.69 \mathrm{~mm}+/-0.67 \mathrm{SD}$ range $(2.5-5 \mathrm{~mm})$. Mean scotopic pupilar diameter was $4.61 \mathrm{~mm}+/-0.78 \mathrm{SD}$ range $(3-6 \mathrm{~mm})$. BPO compound was instilled in both eyes of all participants. One hour after drop instillation, binocular Jaeger was $2.53+/ 1.71$ SD range $(1-6)$ ( $\leq \leq 0.0001)$. Binocular UNVA lines gained were $3.30+/-1.40$ SD range $(0-6)$. Seventy-three percent of participants reached a Jaeger notation of 3 or better, $65.3 \%$ of participants reached a Jaeger notation of 2 or better, and $34.6 \%$ of participants reached a Jaeger notation of 1 . A total of $76.9 \%$ of participants gained 3 or more lines of UNVA, $88.4 \%$ of participants gained 2 or more lines of UNVA, and $96.1 \%$ of participants gained 1 or more lines of UNVA. One patient lost 2 lines of UDVA (3.8\%), and the vision fully recovered after ceasing the effect of the compound; in the rest of the participants, UDVA remained unchanged. The mean photopic pupilar diameter was $2.27 \mathrm{~mm}+/-052 \mathrm{SD}(1.5-3)(\mathrm{p} \leq 0.000)$. Scotopic pupilar diameter was $2.63 \mathrm{~mm}+/-0.53 \mathrm{SD}$ range $(2-3)(\mathrm{p} \leq 0.000)$. All patients were asked to record the time the effect lasted. According to their records, the lasting effect of the composition was 6.17 hours $+/-1.18$ hours SD range (4 to 8 hours). One 52-year-old female patient did not improve any lines of UNVA. Her Jaeger notation was 6 prior to application of the compound and remained 6 one hour after the application. One 53-year-old patient reported nasal congestion grade 5 with an AVS score of 0 to 10 , lasting 15 minutes. Headache was reported in 1 patient (3.8\%). Her pain score was 5 in a 0-10 AVS. The pain lasted less than 10 minutes. One patient reported dim vision that lasted 40 minutes (3.8\%). Hyperemia was scored 0 one hour after instillation in all patients.

\section{Discussion}

In view of current efforts to achieve pharmacological control of presbyopia, it is desirable to reach a compound or compound that improves reasonably well near vision without or with fewer possible collateral effects, such as pain and dim vision, and lasts a reasonable period of time. Our group developed a compound based on low-dose pilocarpine, brimonidine and oxymetazoline combined with sodium hyaluronate and bromofenac to achieve this goal. This compound performs better in improving NUCVA than pilocarpine or brimonidine alone, acting synergically, as we describe in ClinicalTrials.gov identifier NCT05006911. Benozzi reported a combination of pilocarpine (1-2\%) and diclofenac to control presbyopia symptoms. Twenty percent reported eye burning and ocular discomfort after instillation. One percent discontinued the treatment, and $4 \%$ resumed using reading glasses. ${ }^{26}$ In our series, with a lower dose of pilocarpine, pain was reported in $3.8 \%$ of the patients.

Mean baseline Jaeger notation improved from $5.84+/-1.39$ to $2.53+/-1.71(\mathrm{p} \leq 0.0001)$ one hour after the compound instillation, a gain of $3.30+/-1.40$ SD $(0$ to 6). Twenty-five (96.1\%) patients gained one or more lines of UNVA. One patient loss 2 lines (3.8\%). 96.1\% of patients gained at least 1 line of UNVA one hour after drop instillation. Nine (7.6\%) patients did not improve their UNVA with their compound compared with 1 patient (3.8\%) in this study. One patient (3.8\%) presented headache in our study.

The mean baseline photopic pupil diameter was $3.69 \mathrm{~mm}+/-0.67$ (range, 2.5 to 5). One hour after PBO instillation, the pupil diameter was $2.72 \mathrm{~mm}+/-0.52$ (range 2 to 4 ) ( $\leq$ 0.000). The mean baseline scotopic pupil diameter was $4.61 \mathrm{~mm}+/-0.78$ (range, 3 to 6 ). One hour after PBO instillation, the pupil diameter was $2.36 \mathrm{~mm}+/-0.53$ (range 2 to 4$)(p \leq 0.000)$. There was a significant contraction of the pupil due to the combined effect in both photopic and scotopic conditions of this compound.

Giovanna Benozzi reports in 2020 a retrospective series from 1 to 8 years of follow-up of 910 presbyopic individuals. The most frequent side effects were dim vision in 246 individuals (26\%), headache in 119 individuals (12.9\%), and ocular surface burning in 86 individuals (9.3\%). ${ }^{27}$ Although ours is a small population series, one patient reported dim vision (3.8\%), and one patient reported headache (3.8\%). One patient reported no improvement in near vision (3.8\%), and one patient reported hyaline rhinorrhea lasting 30 minutes after drop instillation. Even with a small dose of pilocarpine, adverse reactions can be seen in sensitive individuals. Hyperemia was not reported in any of our patients in this series.

The combination of low-dose pilocarpine, a muscarinic parasympathomimetic, acting on receptors at the iris sphincter and the ciliary muscle, with brimonidine and oxymetazoline at low doses has a synergic effect, improving UNVA one hour after drop instillation. Brimonidine acts at the alpha 2 receptor of the iris dilator muscle, inhibiting its function and inducing indirect miosis. Combining those agents to induce myosis gave us the rationale to lower the concentration to achieve a similar result and avoid side effects such as pain, discomfort and dim vision. We believe that lowering the pilocarpine dose avoids ciliary muscle spasm and related pain and induces myopia, affecting distance vision and eye redness. Only one of our patients reported blurry vision at distance and lost lines of UDVA fully recovering after ceasing the effect of the compound. Oxymetazoline, an alpha 1 and partial alpha 2 imidazolic agonist, has a mydriatic effect. We believe that low concentrations slightly oppose the action of pilocarpine and brimonidine, hence avoiding spasm of the iris sphincter and allowing pupil movement. ${ }^{28}$

This novel combination of pilocarpine, brimonidine and oxymetazoline in low doses has a synergistic effect, improving near vision in presbyopic patients and achieving at least up to 3.73 lines of near vision gain on the Jaeger notation at least one hour after instillation. This compound has the potential to mitigate the effects of presbyopia in healthy individuals. Further studies are being designed to document the long-term effect of this compound in patients with presbyopia.

\section{Conclusion}


The combination of three drugs at low doses, one parasympathetic mimetic (pilocarpine) and two alpha adrenergic drugs (brimonidine alpha 2 selective and oxymetazoline alpha 1 and partial alpha 2 selective), showed a significant synergistic effect, improving near vision, as measured with the Rosembaum chart with Jaeger notation, one hour after instillation in healthy presbyopic patients.

\section{Declarations}

\section{Conflict of interest disclosure}

The author has a patent pending for a Pilocarpine, Brimonidine, Oxymetazoline compound

\section{References}

1. MorganPB,EfronN.Contact lens correction of presbyopia.Cont Lens Anterior Eye.2009;32(4):191-192.

2. StivalLR,FigueiredoMN,SanthiagoMR.Presbyopic Excimer Laser Ablation: A Review.J Refract Surg.2018;34(10):698-710.

3. AlióJL,AmparoF,OrtizD,MorenoL.Corneal multifocality with excimer laser for presbyopia correction.Curr Opin Ophthalmol.2009;20(4):264-271.

4. PallikarisIG,PanagopoulouSI.PresbyLASIK approach for the correction of presbyopia.Curr Opin Ophthalmol.2015;26(4):265-272.

5. ReinsteinDZ,CarpGI,ArcherTJ,GobbeM.LASIK for presbyopia correction in emmetropic patients using aspheric ablation profiles and a micro-monovision protocol with the Carl Zeiss Meditec MEL 80 and VisuMax.J Refract Surg.2012;28(8):531-541.

6. NarooSA,BilkhuPS.Clinical utility of the KAMRA corneal inlay.Clin Ophthalmol.2016;10:913-919.Published 2016 May 18.

7. MoshirfarM,DesautelsJD,WallaceRT,KoenN,HoopesPC.Comparison of FDA safety and efficacy data for KAMRA and Raindrop corneal inlays.Int J Ophthalmol.2017;10(9):1446-1451.Published 2017 Sep 18.

8. MalecazeFJ,GazagneCS,TarrouxMC,GorrandJM.Scleral expansion bands for presbyopia.Ophthalmology.2001;108(12):2165-2171.doi:10.1016/s01616420(01)00591-7

9. ŽiakP,LuckáK,MojžišP,Katuščákovál,HaličkaJ.Prvé skúsenosti s korekciou presbyopie femtosekundovým laserom metódou INTRACOR [First Experience with Femtosecond Laser Presbyopia Correction Method INTRACOR].Cesk Slov Oftalmol.2016;72(3):51-57

10. CummingJS.Performance of the crystalens.J Refract Surg.2006;22(7):633-635.

11. CarsonD,HillWE,HongX,KarakelleM.Optical bench performance of AcrySof(®) IQ ReSTOR(®), AT LISA(®) tri, and FineVision(®) intraocular lenses.Clin Ophthalmol.2014;8:2105-2113.

12. RennaA,AlióJL,VejaranoLF.Pharmacologicaltreatmentsofpresbyopia:areviewofmodernperspectives[publishedcorrectionappearsinEyeVis(Lond).2017Mar2:

13. BenozziG,PerezC,LeiroJ,FacalS,OrmanB.Presbyopia Treatment With Eye Drops: An Eight Year Retrospective Study.Transl Vis Sci Technol.2020;9(7):25.

14. RennaA,VejaranoLF,De laCruzE,AlióJL.Pharmacological Treatment of Presbyopia by Novel Binocularly Instilled Eye Drops: A Pilot Study.Ophthalmol Ther.2016;5(1):63-73.

15. AbdelkaderA,KaufmanHE.Clinical outcomes of combined versus separate carbachol and brimonidine drops in correcting presbyopia.Eye Vis (Lond).2016;3:31.

16. PoinoosawmyD,NagasubramanianS,BrownNA.Effect of pilocarpine on visual acuity and on the dimensions of the cornea and anterior chamber.Br $\mathrm{J}$ Ophthalmol.1976;60(10):676-679.

17. PlaceVA,FisherM,HerbstS,GordonL,MerrillR.Comparative pharmacologic effects of pilocarpine administered to normal subjects by eyedrops or by ocular therapeutic systems.Am J Ophthalmol.1975;80(4):706-712.

18. SmithSE,SmithSA,FriedmannAI,ChastonJM.Comparison of the pupillary, refractive, and hypotensive effects of Ocusert-40 and pilocarpine eyedrops in the treatment of chronic simple glaucoma.Br J Ophthalmol.1979;63(4):228-232.

19. BacskulinA,KundtG,GuthoffR.Efficiencyofpupillarystretchingincataractsurgery.EurJOphthalmol.1998;8(4):230233.CarstoceaB,GafencuO,ArmegioiuM,etal.Dificultaţiînchirurgiacristalinuluilabolnavulvechiglaucomatos[Difficultiesincataractsurgeryofpatientwitholdglaı 39

20. RahmanMQ,RamaeshK,MontgomeryDM.Brimonidine for glaucoma.Expert Opin Drug Saf.2010;9(3):483-491.

21. MundorfT,WilliamsR,WhitcupS,FelixC,BatoosinghA.A3-monthcomparisonofefficacyandsafetyofbrimonidinepurite0.15\%andbrimonidine0.2\%inpatientswithglaucomaorocularhypertension[publishedcorrectionappearsinJOculPharmacolTher.2004Aug;20(4):372].JOc 44.

22. BesadaE,ReedK,NajmanP,ShechtmanD,HardiganP.Pupillometry study of brimonidine tartrate $0.2 \%$ and apraclonidine $0.5 \%$.J Clin Pharmacol.2011;51(12):1690-1695.

23. KeslerA,ShemeshG,RothkoffL,LazarM.Effect of brimonidine tartrate 0.2\% ophthalmic solution on pupil size.J Cataract Refract Surg.2004;30(8):17071710.

24. LeeJH,YouYS,ChoeCM,LeeES.Efficacy of brimonidine tartrate $0.2 \%$ ophthalmic solution in reducing halos after laser in situ keratomileusis.J Cataract Refract Surg.2008;34(6):963-967.

25. FuderH.Functional consequences of prejunctional receptor activation or blockade in the iris.J Ocul Pharmacol.1994;10(1):109-123.

26. Jorge Benozzi Presbyopia:a New Potential Pharmacological Treatment MEHDIOphthalmology Journal2012;Vol.1,No1 
27. BenozziG,PerezC,LeiroJ,FacalS,OrmanB.Presbyopia Treatment With Eye Drops: An Eight Year Retrospective Study.Transl Vis Sci Technol.2020;9(7):25.Published 2020 Jun 23.doi:10.1167/tvst.9.7.25

28. ChuTC,OgidigbenMJ,PotterDE.Oxymetazoline: potential mechanisms of inhibitory effects on aqueous humor dynamics.Pharmacology.1996;53(4):259270.doi:10.1159/000139438 